\title{
A MODEL FOR THE DESCRIPTION OF THE INFORMATION
}

SYSTEM DYNAMICS

\author{
A. FLORY, J. ROULOUMDIAN \\ Laboratoire d'informatique \\ Université Claude Bernard - LYoN
}

This paper deals with the description of the information system dynamics at the conceptual level. A model is put forward to take into account both the structural and dynamic aspects of data base. The data model (an entity-relation model) helps to build the data into a structured set of relations that correspond to a specific third normal form. These relations do not play the same role in data base evolution : we propose a classification of "event" relations - the tuples of which are the starting point of treatments - and of "permanent" relations. The description of processings deriving from a primary event may be divided into elementary chronological steps with the help of a hierarchy graph. Each step can be analyzed in term of "states" which express the stage of treatment of the triggering event.

\section{$1-$ INIRODUCTION :}

Nowadays, it is agreed by most people that the design of a data base must begin with the definition of a conceptual schema wich "represents the enterprise's view of the structure it is attempting to model in the data base" (ANS 75).

This conceptual schema is derived from a model which defines the correse pondence between the data stored in the base and the facts of the real world which are of interest for the organization. The symbolic representation of these facts can be considered from both a static point of view (description of the real world objects and of their mutual relationship) and a dynamic one (description of the data base modifications induced from the insertion of a new fact). Thus sone authors (HUI 76) speak of a "completude criterion" of the model, which indicates its ability to take into account the characteristics of the real world. 
A lot of models have been put forward mainly in the field of the relational models. One advantage of this type of models is to keep the conceptual schema free from implementation aspects. Since their first description (COD 70), these models have been studied into 2 directions : first the analysis of their mathematical properties (for example : research of the minimal cover of a set of functional relations, decomposition and normalization processes), second, their semantic purport because fact representation is not very easy with such mathematical models. For this reason, entity-relation models were created (CHE 76).

The introduction of the dynamic components of information systems at the conceptual schema level can take several shapes in scientific literature : Association of integrity constraints to the data model (MAC 76) (HAM 76) (ESW 75) (WEB 76), use of high level procedural languages (HUI 76) or definition of a set of "evolution rules" triggered by "events" (BEN 76).

However it seems to us that none of these models clearly shows the link which exists between the stored data and its evolution deriving from the storage of a new fact. The aim of this paper is to describe a model which includes both structural and dynamic aspects. In our model which belongs to the entity-relation type :

a) every real world entity of interest for the organization, appears as a third normal form relation tuple in the data base.

b) therefore when a new event (entity) is taken into account in the organization, modifications of the data base can be expressed very easily as tuple updatings or creations.

The next paragraph concerns a short description of the part of the model which deals with the structure of data. A more complete presentation is given in (FLO 78). In paragraph 3, a classification of the relations which represent the entity types of the real world is proposed according to the type of the domains composing these relations. In this typology, the relations are splitted into two classes : first the relations representing events, called "event" relations. The tuples of such relations can trigger procedures which event relations refer but which are not the starting point of treatment. Finally, in paragraph 4, all the concepts needed for the description of the dynamics of the information system are introduced.

2 - STRUCTURAL COMPONENTS OF THE MODEL : 
In our model the data structure is expressed as a set of relations each of which is composed of a set of elementary attributes. In each relation, a subset of attributes (possibly one attribute) plays a key role, that is to say that the other attributes of the set are functionally dependant of this subset. The attributes that do not belong to the key are called characteristics of the relation.

Each relation is in third nomal form (COD 72) and is called an entitytype (denoted E.T. later on).

Two kinds of E.T.'s can be distinguished :

- first order E.T.'s the key of which includes a singie attribute. These E.T.'s are images of object types of the real world. 1-order E.T. characteristics express the properties of these objects. For example the E.T. "ITEM" (the key is underlined)

$$
\text { (ITEM \# , PRICE, NAME, QTY-ON-HAND) }
$$

corresponds to the products manufactured in the enterprise.

- n-order E.T.'s the key of which is composed of more than one attribute. These E.T.'S depict the relation existing between two or more 1-order E.T.'s. For instance the n-order E.T. "ORDER-LINE" :

(OROER * ITTH \#, ORDERED-CIY)

expresses a relation between the 1-Order E.T. "ORDER" and "ITIN" which have respectively ORDER \# and ITPM \# as key.

Iet $C$ be the set of all the attributes which occur in the information system and $S$ the set of the E.T.'s formed on C.

$S$ is called an entity type system (noted E.T.S. later on) if the following conditions are satisfied :

1) Each attribute of $C$ belongs at least to one E.T.,

2) If an attribute of $C$ belongs to several E.T.'s,

- either it plays a key role in one of the E.T.'s

- or it cannot appear in the righ side of a functional relations.

An example of an E.T.S. is given below (FIG 1). It has five E.T.'s called 
"DEITV-SLIP", "ORD", "CUST", "ITEM" and "REPR" (corresponding respectively to the object type DELIVERY-SLIP, ORDER, CUSTORER, ITEM and REPRESENTATIVE of the real world).

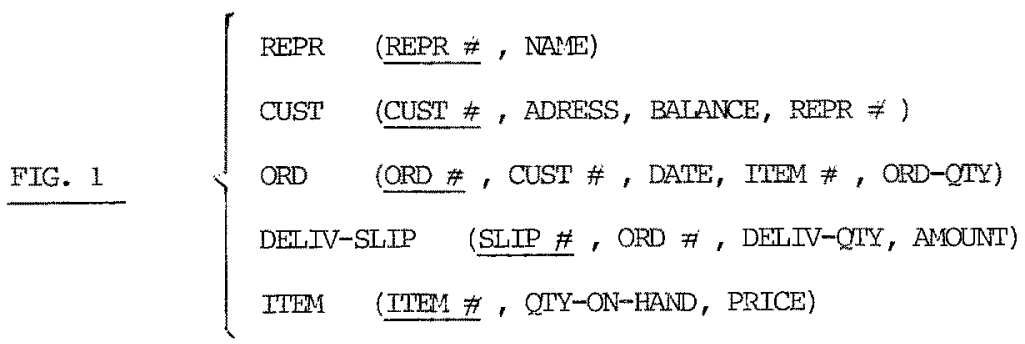

(we suppose there is one order line for each order).

Using an arrow to represent a functional relation (noted F.R. later on) between two attributes, this data structure can be visualized as follows (FIG. 2) (each rectangle represents a E.T.).

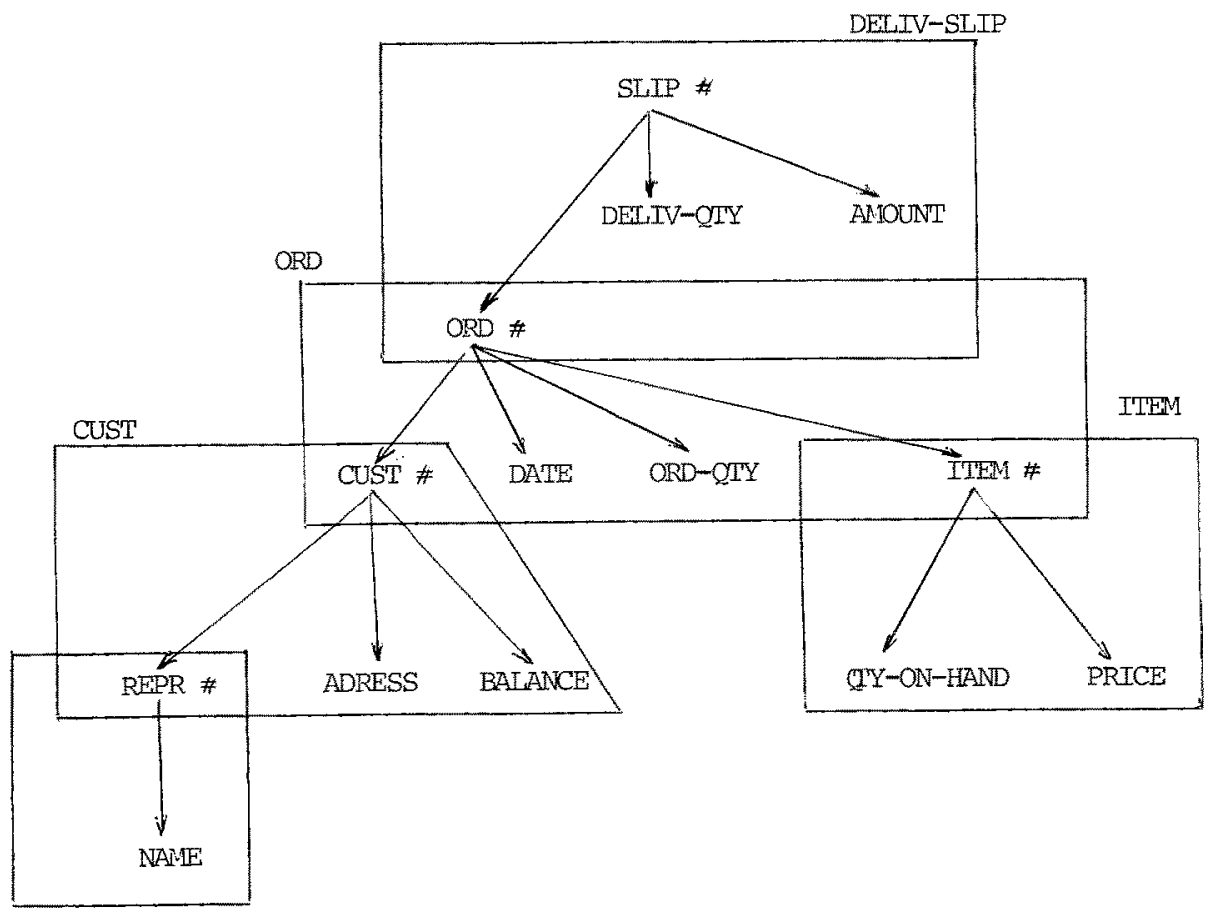

REPR

FIG. 2 
On this graph we can see that the E.T.'s are linked together through the F.R. existing between their keys (An attribute which occurs in more than one E.T. mast necessarily be the key in one of the E.T.'S). So the F.R.' $s$ are defined either inter E.T.'s (e.g. ORD \# $\rightarrow$ CUST $\#$ ) or intra an E.T. (e.g. CUST $\# \rightarrow$ ADRESS).

Algorithms leading to the E.T.S. are given in (FLO 77). In a conceptual schema, the E.T.S. Is shown to be unique.

In order to define the role played by the E.T.' $s$ in the dynamics of the information system, we must classify the attributes according to two criteria : first, the way they appear in the system (calculated by the information system, or given by the environment) ; second, the stability of their values in the time. By combining the first criterion (calculated/not calculated) and the second one (stable/unstable), we can define four classes of attributes. Thus, for the afore mentioned example, we have the following classification.

\begin{tabular}{l|l|l} 
& stable & unstable \\
\hline \multirow{3}{*}{ calculated } & $\begin{array}{l}\text { SLIF \# } \\
\text { DEIIV-CIY } \\
\text { AMOUN }\end{array}$ & $\begin{array}{l}\text { BALANCE } \\
\text { OTY-ON-HAND }\end{array}$ \\
\hline & CUST \# & \\
not calculated & DAIE & ADRESS \\
(given) & ORD-QIY & PRICE \\
& ORD \# & NAME \\
& ITEM \# & \\
REPR \# &
\end{tabular}

The definition of the data structure can be viewed at two different levels : A "detail" level in wich the description includes all the attributes (as in FIG. 1), and a "macroscopic" level which handles only the E.T. keys and inter E.T. functional relations. At this level, to a set of F.R.'s there corresponds a graph which defines a partial order on the keys, which we call key hierarchy. In our exemple, the hierarchy will be (FIG. 3)

FIG. 3

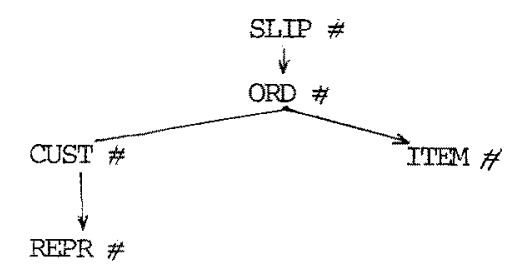


This hierarchy is quite valuable for the description of the information system dynamics.

3 - IOGICAL AND CHRONOLOGICAL F.R.'s :

\section{1. - Classification of the entity types :}

The informations to be menorized belong to two classes : they refer either to the "living" objects of the real world or to the manifestations of these objects. The properties of the first class of objects can change as time elapses : for instance the address of a customer or the surface of a room. On the contrary, the manifestations of "living" objects do not change and are (explicitly or implicitly) dated. They correspond to the notion of events : for instance, a hotel reservation is an event referring to the "living" objects "person" and "hotel". A date is associated to a reservation and any modification of the reservation characteristics defines a new event which deletes the previous reservation.

We think that in the information system the most important part of the dynamics is inferred from the events as defined above. It is therefore important to be able to distinguish them in the data base. This can be done by analyzing the type of the E.T. attributes.

Calculated-unstable attributes are state variables, which are introduced to facilitate the date base management but they are not essential for the description of the data base. Indeed, the present state of a data base can be deduced from its initial state and from the set of events which occured since the beginning. For that reason, we call these attributes "situational".

Thus that kind of attributes is not helpful if one wants to differentiate the E.T.'s we will rather use the three other kinds of attributes to define a typology of the E.T.'s which is useful in the description of the dynamics :

An E.T. will be called "external event" if all its attributes (other than situational) are stable and not calculated (for example the E.T. "ORDER").

An E.T. will be called "internal event" if all its attributes (other than situational) are stable and calculated (for instance "DEITV-SLIP").

An E.T. Which is neither "external" nor "internal event" is called permanent (in our example CUST, ORD and ITEM). One or more of their attributes must be unstable- not calculated. 
Thus we define an "event" as a tuple of a relation whose value does not change as time alapses and which may have several different "states", as will be seen later on. We suppose that its life time, before it is archived, is short. Its insertion, in a relation may start a procedure of the information system. On the other hand, the permanent E.T. tuples have a longer life time and are referred to by the events.

\section{2. - F.R. semantics :}

The semantics of an "inter" E.T. functional relation are different according to the types of the E.T.'s involved in F.R.. We will perhaps give some definitions to begin with :

A F.R., $A \longrightarrow B$, will be called strong if it is defined for each value of $A$. It will be called stable if it is always the same values of $B$ which is associated to a given value of A. Finally, a F.R. will be total if it is both strong and stable.

Given two E.T.'S $X$ and $Y$, a total relationship between their keys may have two different meanings : first it may mean that an entity of type $X$ is an emanation of an entity of type $Y$. For instance : class-room $\# \longrightarrow$ school \#. In this case, the F.R. will be called logical.

Second, it may indicate that there exists a chronology in the creation of the two entities. For example the total R.F.

\section{INVOICE $\# \rightarrow$ ORDER $\#$}

implies that the INVOICE is createdafter the order.

If a chronological functional dependency exists between two E.T. keys, then at least one of the E.T.'s belongs to the event type. On the contrary, a F.R. between two permanent E.T. keys expresses a logical relationship.

For example, let us consider the key hierarchy given in figure 4 .

Logical and chronological relationship is noted respectively I and c. From this very simple example, we can note that the primary event is the order and that its treatment involves two steps : first, the creation of a delivery slip which then leads to an invoice. 


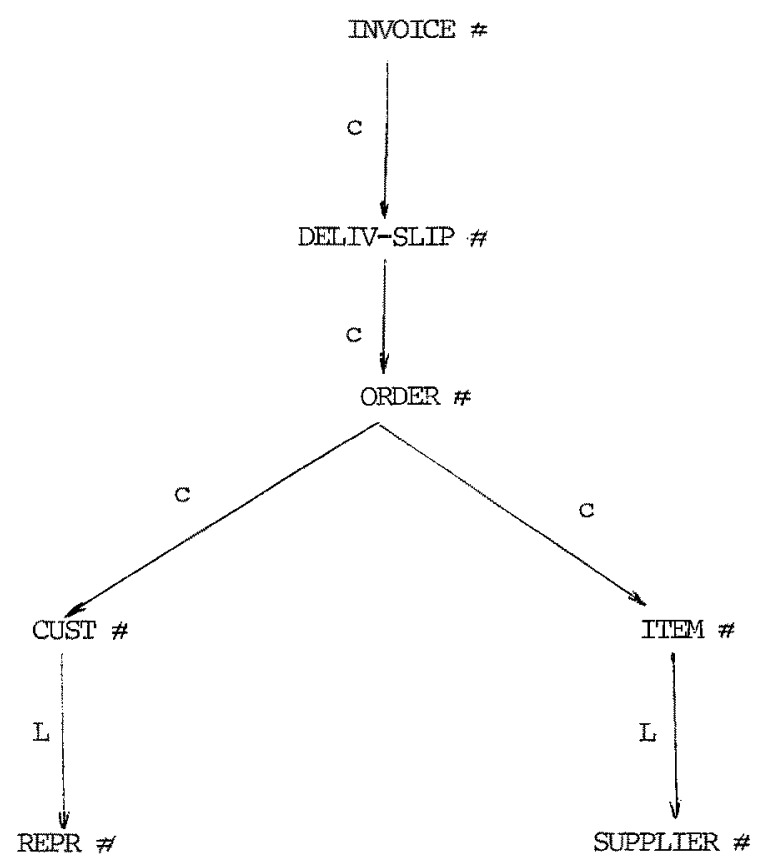

Figure 4.

In some way, and with the help of the key hierarchy we will be able to define the "gross architecture" of the treatments associated to a primary event. Though a few precautions are needed when the hierarchy is handled in order to get the processing sequence, the notion of chronology seems a useful tool in the analysis of an information system.

The next paragraph will deal with the concepts used in the description of the treatment of an elementary step (e.g. order $\rightarrow$ delivery slip or delivery slip $\longrightarrow$ invoice) .

4 - DESCRIPTION OF INEORMATION SYSTEM DYNAMICS :

Similarly to several authors (HAM 76) (ESW 75) (BEN 76) we will assume that for the description of information system dynamics, one must define the valid states of the data base and the allowed transitions between these states. Before we delineate what a data base state is, let us notice that in this paper we only consider individual processing, that is to say the processing resulting from a new 
fact ( event) collected in the data base. We do not take into account set processings (such as statistical treatments) starting from an external decision.

\section{1. - Tuple state in an E.T. :}

On way of indicating at which stage of processing a given tuple is in an E.T., is to define the concept of tuple state. This notion must be define previous to the definition of data base state.

For instance let us consider a tuple of the E.T. "ORD" : we are not able to say if it concerns an order which has already been processed or a new order. So we will define a tuple state as an information associated to each tuple of a E.T. and which may have a finite number of values. For instance, in the management of an order, one can define four valid states :

State 0 : registered order

State 1 : refused order

State 2 : waiting order

State 3 : fulfilled order

Processing an order is equivalent to realizing a transition between two of the above described states. Only certain transitions are allowed. A graph can be used for the representation of these transitions (figure 5). In this graph the number between parenthesis indicates the state.

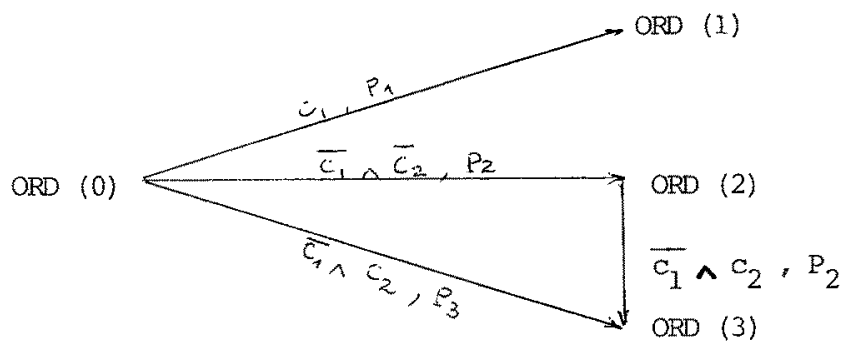

Figure 5.

The existence of an arc between the nodes $i$ and $j$ of this graph means that a given tuple of the E.T. "ORD", in state $i$, may be processed to state $j$ by 
the procedure $P_{i j}$ provided that the predicate $C_{i j}$ is fulfilled.

In our example, we consider two predicates which can be expressed under the following form (we assume that $X, Y, Z, T$ are tuples respectively of CuST, $\mathrm{ORD}(0)$, ORD (0) U ORD (2) and ITEM).

$$
\begin{aligned}
& C_{1}: x . \text { CUST \# }=\text { Y.CUST \# } \wedge \text {. BAIANCE }>\text { given value } \\
& C_{2}: \text { Z.ITEM } \#=\text { T. ITEM }=\wedge \quad \text { X. ORD-QIY } \leqslant \text { T.QTY-ON-HAND. }
\end{aligned}
$$

So the set of the E.T. "ORD" is partitioned into four equivalence classes the population of which will have the same processing.

In figure 5, states 1 and 3 are final states and refer to completely processed information (information to archive or delete).

\section{2. - Description of the information system dynamics :}

Because of the correspondence between the objects of the real world and the entity types of our data model, the dynamics can be described in a simple way, for in this case the tuple is a "natural" manipulation unit :

Once more, let us take our example and suppose that a new order is inserted (in state 0), which satisfies the predicate $\vec{c}_{1} \wedge c_{2}$ (figure 5). The procedure $P_{3}$ involves :

- the updating of the quantity-on-hand corresponding to the ordered item

- the creation of a tuple in the E.T. DEITV-SLIP according to the calculation rules of its attributes.

- the change of the handled order to state 3.

The created DELIV-SLIP may in turn start a procedure leading to the tuple insertion corresponding to the creation of an invoice. In this example we can see that a primary external event (an order) may be handied by a procedure which created a tuple (in DELIV-SLIP) : this tuple appears then as a (secondary) internal event.

Thus the dynamics are expressed in term of process synchronization, each process been associated to an "event" which is a tuple creation in our model. 
Though the purpose of this paper is only to give some concepts which seem to us, rather useful, it appears that a high level set oriented language would be very convenient in this approach of the information system dynamics.

\section{3. - Data base state :}

The data base state is defined by the set of the tuple states in the various E.T.'s ; the data base state changes when a transition between two states of a given tuple takes place. As seen above the state changement may involve situationel attribute updating, tuple creation or deletion.

The data base is in a stable state if all the tuples of "event" E.T.'s are either in final states or in states for which no predicate is satisfied. A change in the data base can then occur only if an external event appears.

5 - CONCLUSTON :

Though up to now, very few papers have linked data structure and the description of dynamics at a conceptual level, it seems to us that these two con ponents are strongly dependent on each other. It is according to the data model that a more or less convenient description of the evolution of the data base will be reached. our model is an entity-relation one. It introduces in a simple way the notion of event commonly used in literature but seldom clearly defined. Thus the resulting data structure is in an "optimal third normal form" as called by SCHMIOT (SCH75). In the data base, relations play either "event"or "permanent" role. In most cases, we think that the event role can be deduced from the type of relation attributes. The processing inferred from a primary external event can be broken down into a succession of elementary steps each of which is characterized by a state graph.

It appears that the concept of state is a common one with data users and thus it may be a useful tool in the analysis of an information system. 
(ANS 75) ANSI/X3/SPARC interim report (1975)

(BEN 76) BENCI G., BODART F., BOCAERT H, CABANES A. : "Concept for the design of a conceptual schema". Modelling in D.B.M.S. North Holl. PUBL. (1976) pp. $181-200$.

(CHE 76) CHEN P. : "the entity-relationship model : toward a unified view of data" A.C.M. Transactions on data base systems. Vol.1 $n^{\circ} 1$ (march 1976) pp 9-36.

(COD 70) CODD E.F. : "A relational model of data for large shared data banks" Con. A.C.M. 13 (June 1970) pp. 337-387.

(COD 72) CODD E.F. : "Further normalization of the data base relational model" Current Computer Schience Symposia, Data Base System Vol. 6 prentice Hall (1971)

(ESW 75) ESWRRAN P., CHANBERLIN P. : "Functional specifications of a subsystem for data base integrity" Proc. Very Large Data Base Conf. (Sept. 1975) pp. $48-68$

(FLO 77) FLORY A. : "Un modèle et une méthode pour la conception logique d'une base de données" Ph. Thesis (nov. 1977).

(FLO 78) ELORY A., KOULOUMDJIAN J. : "A model and a method for logical data base design" Very Large Data base conf. West Berlin Sept 1978 pp. $315-326$.

(HAM 75) HAMIAR M., MC IEOD J. : "Semantic Integrity in a relational data base systen" Proc. Very Large Data Base Conf. (Sept. 1975) pp. 25-47.

(HUI 76) HUITS M.H.M. : "Requirements for languages in data base systems" DATA Base Description - North-Holland pub. (1975) pp. 85-110.

(MAC 76) MAC GHEEIS $C$. : "A procedural language for expressing integrity constrainsts in the coexistence model" Modelling in D.B.M.S. North-Holland Pub. (1976) pp. 293-301.

(SCH 75) SCHMD H.A., SWENSON J. : "On the semantics of the relational data model" A.C.M. STGMod Conf. on managt of data (nai 1975).

(WEB 76) WEBER H. : "A semantic model of integrity constraints on a relational data base". Modelling in D.B.M.S. North-Holland Pub. (1976) pp. 269-292. 